\title{
Synthesis and Evaluation of Benzoquinolinone Derivatives as SARS-CoV 3CL Protease Inhibitors
}

\author{
Tae-Young Ahn, ${ }^{\dagger,}$ Chih-Jung Kuo,${ }^{\S, \#}$ Hun-Ge Liu, ${ }^{\S}$ Deok-Chan Ha, ${ }^{\ddagger}$ Po-Huang Liang, ${ }^{\S, \#}$ and Young-Sik Jung ${ }^{\dagger, *}$ \\ ${ }^{\dagger}$ Bio-organic Science Division, Korea Research Institute of Chemical Technology, Daejeon 305-600, Korea \\ *E-mail: ysjung@krict.re.kr \\ ${ }^{\ddagger}$ Department of Chemistry, Korea University, Seoul 136-701, Korea \\ ${ }^{\S}$ Institute of Biological Chemistry, Academia Sinica, Taipei 11529, Taiwan R.O.C. \\ \#Taiwan International Graduate Program, Academia Sinica, Taipei 11529, Taiwan \\ Received September 11, 2009, Accepted November 18, 2009
}

\begin{abstract}
For the discovery of new antivirals against Severe Acute Respiratory Syndrome-coronavirus (SARS-CoV), we prepared and evaluated several benzoquinoline compounds as its 3C-like protease (3CLpro) inhibitors. Based on the computer modeling study that each of the two rigid benzoquinolinone and N-phenoltetrazole moieties of the compound $\mathbf{1}$ is bound to the S1 and S2 sites, respectively, of the SARS protease by forming H-bonds and hydrophobic interactions, we designed and synthesized alkylated benzoquinolines at both the sites of the hydroxyl groups. We found that the compound 2a showed five times higher inhibiting activity against the 3CLpro compared to the compound 1.
\end{abstract}

Key Words: Benzoquinoline, Coronavirus, Picornavirus, 3C protease, Computer modeling

\section{Introduction}

Coronaviruses $(\mathrm{CoV})$ are the positive-stranded RNA viruses with largegenome of 27 - $32 \mathrm{~kb}$, which typically cause respiratory and enteric diseases, pneumonia, exacerbation of asthma, neurological symptoms and myocarditis in humans and domestic animals. An outbreak of severe acute respiratory syndrome (SARS), caused by a novel human $\mathrm{CoV}$, was spread from China to 29 countries in 2003 , infecting a total of $\sim 8,000$ people and killing $\sim 800$ patients. ${ }^{1}$ SARS-CoV contains a $3 \mathrm{C}$-like protease $\left(3 \mathrm{CL}^{\mathrm{pro}}\right.$ ) analogous to the $3 \mathrm{C}^{\text {pro }}$ of picornaviruses (PV), which responsible for processing two overlapping polyproteins, ppla (486 kDa) and pplab (790 kDa). Other members of human CoV including CoV-229E, CoV-OC43, CoV-HKU1 and CoV-NL63 also require a $3 \mathrm{CL}^{\text {pro }}$ for the maturation of viral proteins. Several inhibitors have been developed to inhibit the $3 \mathrm{CL}^{\text {pro }}$ of SARS$\mathrm{CoV}^{2}$ In our previous studies, ${ }^{3}$ we performed high throughput

\section{1}

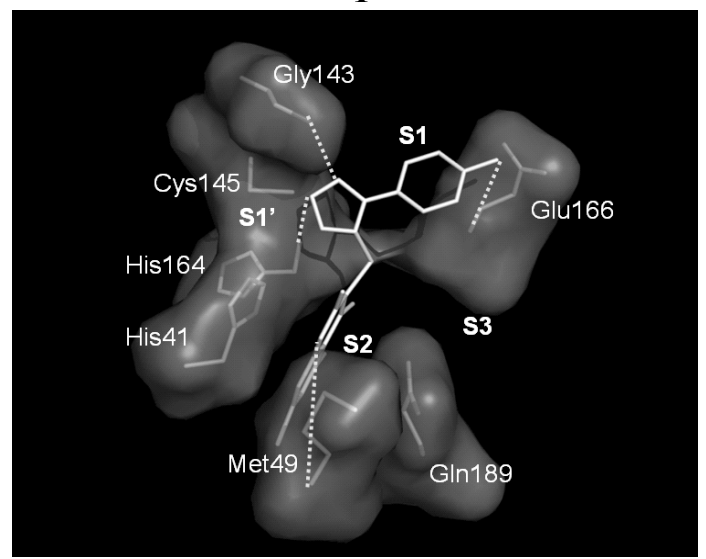

screening using a library of $\sim 6800$ compounds to find five novel inhibitors of the SARS-CoV $3 \mathrm{CL}^{\text {pro }}$ and these also inhibited another human CoV-229E 3CL ${ }^{\text {pro }}$. Among the five inhibitors, the compound 1 (structure shown in Figure 1) showed $\mathrm{IC}_{50}$ values of $10.6 \mu \mathrm{M}$ and $12.4 \mu \mathrm{M}$, respectively, against SARS 3CL ${ }^{\text {pro }}$ and $229 \mathrm{E} 3 \mathrm{CL}^{\text {pro }}$. This compound is a competitive inhibitor with

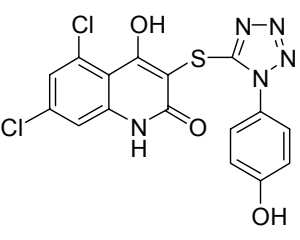

1

Figure 1. Structure of Benzoquinolinones

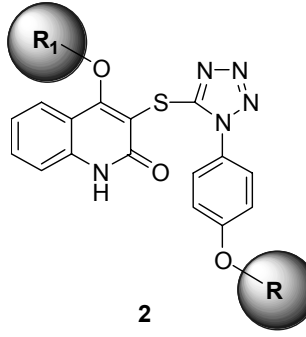

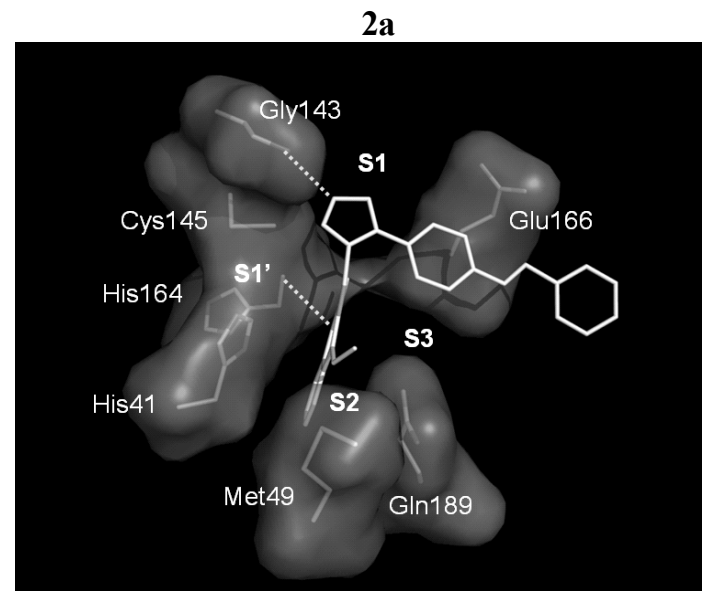

Figure 2. Computer Modeling of the Binding Modes of the Benzoquinoline $\mathbf{1}$ and 2a at the Active Site of the SARS $3 \mathrm{CL}^{\text {pro }}$. 
respect to the substrate (data not shown), indicating that it binds in the active site. This compound can be considered as two rigid benzoquinolinone and $\mathrm{N}$-phenoltetrazole moieties, which are connected by sulfur atom. Based on the computer modeling, each of these aromatic moieties is bound to the S1 and S2 site of the SARS protease by forming H-bonds and hydrophobic interactions, respectively. ${ }^{3}$ This binding mode indicates that there are extra rooms around the two hydroxyl groups of the compound. Therefore we aimed to introduce hydrophobic groups on the hydroxyl groups of compound $\mathbf{1}$.

From the previous studies, ${ }^{3}$ we also found hits which not only inhibited $3 \mathrm{CL}^{\text {pro }}$ from SARS-CoV but also inhibited 3Cpro from PV. Picornaviruses are small nonenveloped RNA viruses with a single strand of genomic RNA of 7500 - 8000 nucleotides. ${ }^{4}$ The members of PV include rhinoviruses (RV), enteroviruses $(\mathrm{EV})$, coxsackieviruses $(\mathrm{CV})$, polioviruses, echoviruses, encephalomyocarditis viruses, meningitis virus, foot and mouth viruses, hepatitis A virus, and so on. Among them, EV and CV infection can cause hand, foot, and mouth diseases in humans and animals. In these PV, a chymotrypsin-like protease (named $3 \mathrm{C}^{\mathrm{pro}}$ ) is required to process polyproteins into mature proteins for viral replication. ${ }^{5}$ Herein we report the synthesis of some benzoquinoline derivatives and test their inhibitory activities on 3Cpro from EV71 and CVB3 and 3CLpro from SARS-CoV. One compound shows improved activity against 3CLpro of SARSCoV.

\section{Materials and Methods}

Expression and purification of the proteases. Two types of proteases including $3 \mathrm{CL}^{\text {pro }}$ from SARS-CoV and $3 \mathrm{C}^{\text {pro }}$ from CVB3 and EV71 were used to assay the inhibitors in this study. The $3 \mathrm{CL}^{\text {pro }}$ from SARS-CoV and $3 \mathrm{C}^{\text {pro }}$ from EV71 and CVB3 were prepared as reported previously. ${ }^{6}$

Synthesis of the compounds. All reactions were carried out under $\mathrm{N}_{2}$ atmosphere unless otherwise noted. Tetrahydrofuran (THF) was distilled over $\mathrm{Na}$ and $\mathrm{CH}_{2} \mathrm{Cl}_{2}$ was distilled over $\mathrm{CaH}_{2}$ prior to use. Organic extracts or filtrates were washed with brine, dried over anhydrous $\mathrm{Na}_{2} \mathrm{SO}_{4}$ and concentrated in vacuo. Flash chromatography was performed with Merck-EM Type 60 (230 400 mesh) silica gel. ${ }^{1} \mathrm{H}$ NMR and ${ }^{13} \mathrm{C}$ NMR spectra were measured on Bruker $300 \mathrm{MHz}$ spectrometer. Mass spectrometric data were determined by use of Varian 1200L Gas Chromatograph/Mass Spectrometer. IR spectrometric data were determined by use of ATR-FRIR Spectrometer. Melting points are uncorrected.

Methyl 2-(2-chloroacetamido)benzoate (4): To a solution of methyl 2-aminobenzoate $3(10.4 \mathrm{~g}, 68.8 \mathrm{mmol})$ in $\mathrm{CH}_{2} \mathrm{Cl}_{2}(300$ $\mathrm{mL})$ was added $\mathrm{Et}_{3} \mathrm{~N}(19 \mathrm{~mL}, 0.13 \mathrm{~mol})$ and chloroacetyl chloride $(6.6 \mathrm{~mL}, 82 \mathrm{mmol})$, and the mixture was stirred at $\mathrm{rt}$ for $1 \mathrm{~h}$. The mixture was diluted with $\mathrm{CH}_{2} \mathrm{Cl}_{2}$, and then washed with $1 \mathrm{~N} \mathrm{HCl}$ followed by brine. Concentration of the organic solution gave $4(14.0 \mathrm{~g}, 90 \%)$ as a white solid. mp: $215-218^{\circ} \mathrm{C} ;{ }^{1} \mathrm{H}$ NMR $\left(300 \mathrm{MHz}, \mathrm{CDCl}_{3}\right) \delta 3.92(\mathrm{~s}, 3 \mathrm{H}), 4.21(\mathrm{~s}, 2 \mathrm{H}), 7.12-7.17(\mathrm{~m}$, $1 \mathrm{H}), 7.53-7.59(\mathrm{~m}, 1 \mathrm{H}), 8.06(\mathrm{dd}, 1 \mathrm{H}, J=1.6 \mathrm{~Hz}, 8.0 \mathrm{~Hz}), 8.70$ $(\mathrm{dd}, 1 \mathrm{H}, J=1.0 \mathrm{~Hz}, 8.5 \mathrm{~Hz}), 11.84(\mathrm{~s}, 1 \mathrm{H})$.

Methyl 2-\{2-[1-(4-hydroxyphenyl)-1 H-tetrazol-5-ylthio] acetamido $\}$ benzoate (6): To a solution of methyl 2-(2-chloro- acetamido)benzoate $4(6.00 \mathrm{~g}, 26.4 \mathrm{mmol})$ in THF $(100 \mathrm{~mL})$ was added $\mathrm{Et}_{3} \mathrm{~N}$ (4.0 mL, $\left.29 \mathrm{mmol}\right)$ and 4-(5-mercaptotetrazol-1-yl) phenol 5 (5.60 g, $29.0 \mathrm{mmol})$, and the mixture was heated at 80 ${ }^{\circ} \mathrm{C}$ for $3 \mathrm{~h}$. The mixture was concentrated, diluted with $\mathrm{CH}_{2} \mathrm{Cl}_{2}$, and washed with $5 \% \mathrm{NaHCO}_{3}$. The crude was purified by column chromatography (ethyl acetate : hexane $=1: 5$ ) to give 6 $(0.14 \mathrm{~g}, 27 \%)$ as a white solid. $\mathrm{mp}: 170-173{ }^{\circ} \mathrm{C} ;{ }^{1} \mathrm{H}$ NMR $(300$ $\left.\mathrm{MHz} \mathrm{CDCl}_{3}\right) \delta 3.89(\mathrm{~s}, 3 \mathrm{H}), 4.34(\mathrm{~s}, 2 \mathrm{H}), 7.00(\mathrm{~d}, 2 \mathrm{H}, J=8.7$ $\mathrm{Hz}), 7.10-7.15(\mathrm{~m}, 1 \mathrm{H}), 7.49-7.58(\mathrm{~m}, 3 \mathrm{H}), 8.01(\mathrm{dd}, 1 \mathrm{H}, J=1.3$ $\mathrm{Hz}, 7.9 \mathrm{~Hz}), 8.63$ (d, 1H, $J=8.4 \mathrm{~Hz}), 11.12$ (s, 1H).

Methyl 2-(2-\{1-[4-(benzyloxy)phenyl]-1H-tetrazol-5-ylthio\} acetamido)benzoate (7a): To a solution of methyl 2-\{2-[1(4-hydroxyphenyl)-1 $H$-tetrazol-5-ylthio]acetamido $\}$ benzoate $6(0.50 \mathrm{~g}, 1.30 \mathrm{mmol})$ in DMF $(5 \mathrm{~mL})$ was added $\mathrm{K}_{2} \mathrm{CO}_{3}(0.27$ $\mathrm{g}, 1.9 \mathrm{mmol})$ and benzyl bromide $(0.23 \mathrm{~mL}, 1.9 \mathrm{mmol})$, and the mixture was stirred at $\mathrm{rt}$ for $3 \mathrm{~h}$. The mixture was extracted with ethyl acetate, and then purified by column chromatography (ethyl acetate : hexane $=1: 2)$ to give $7 \mathbf{a}(0.50 \mathrm{~g}, 82 \%)$ as a white solid. mp : $125-128{ }^{\circ} \mathrm{C} ;{ }^{1} \mathrm{H}$ NMR $\left(300 \mathrm{MHz}, \mathrm{CDCl}_{3}\right) \delta 3.87$ (s, $3 \mathrm{H}), 4.33(\mathrm{~s}, 2 \mathrm{H}), 5.14(\mathrm{~s}, 2 \mathrm{H}), 7.09-7.16(\mathrm{~m}, 3 \mathrm{H}), 7.35-7.46(\mathrm{~m}$, $5 \mathrm{H}), 7.51-7.58(\mathrm{~m}, 3 \mathrm{H}), 8.00(\mathrm{dd}, 1 \mathrm{H}, J=1.6 \mathrm{~Hz}, 8.0 \mathrm{~Hz}), 8.64$ (d, $1 \mathrm{H}, J=8.4 \mathrm{~Hz}), 11.64(\mathrm{~s}, 1 \mathrm{H})$.

Methyl 2-\{2-[1-(4-ethoxyphenyl)-1 $\boldsymbol{H}$-tetrazol-5-ylthio]acetamido\} benzoate (7b): white solid, $\mathrm{mp}: 130-132{ }^{\circ} \mathrm{C} ;{ }^{1} \mathrm{H} \mathrm{NMR}$ $\left(300 \mathrm{MHz}, \mathrm{CDCl}_{3}\right) \delta 1.33(\mathrm{t}, 3 \mathrm{H}, J=7.4 \mathrm{~Hz}), 3.88(\mathrm{~s}, 3 \mathrm{H}), 4.04$ (q, $2 \mathrm{H}, J=7.5 \mathrm{~Hz}), 4.32(\mathrm{~s}, 2 \mathrm{H}), 7.11(\mathrm{~d}, 2 \mathrm{H}, J=8.9 \mathrm{~Hz}), 7.19-$ $7.24(\mathrm{~m}, 1 \mathrm{H}), 7.30(\mathrm{~d}, 1 \mathrm{H}, J=8.2 \mathrm{~Hz}), 7.57-7.63(\mathrm{~m}, 3 \mathrm{H}), 7.92$ $(\mathrm{d}, 1 \mathrm{H}, J=7.8 \mathrm{~Hz}), 11.65(\mathrm{~s}, 1 \mathrm{H})$.

Methyl 2-\{2-[1-(4-methoxyphenyl)-1H-tetrazol-5-ylthio] acetamido\} benzoate (7c): white solid, $\mathrm{mp}: 142-146{ }^{\circ} \mathrm{C} ;{ }^{1} \mathrm{H}$ NMR $\left(300 \mathrm{MHz}, \mathrm{CDCl}_{3}\right) \delta 3.88(\mathrm{~s}, 3 \mathrm{H}), 3.89$ (s, 3H), 4.32 (s, 2H), 7.04-7.14 (m, 3H), 7.51-7.58 (m, 3H), 8.00 (dd, $1 \mathrm{H}, J=1.5$ $\mathrm{Hz}, 8.0 \mathrm{~Hz}), 8.64$ (d, 1H, J=8.4 Hz), 11.64 (s, 1H).

3-[1-(4-Benzyloxyphenyl)-1H-tetrazol-5-ylthio]-4-hydroxyquinolin-2-(1H)one (8a): To a solution of HMDS $(0.67 \mathrm{~mL}$, $3.2 \mathrm{mmol})$ in THF $(10 \mathrm{~mL})$ was added $1.6 \mathrm{M}$ of $n$-BuLi $(2.0 \mathrm{~mL}$, $3.2 \mathrm{mmol}$ ) at $-78^{\circ} \mathrm{C}$, and the mixture was stirred for $1 \mathrm{~h}$. To a solution of methyl 2-(2-\{1-[4-(benzyloxy)phenyl]-1H-tetrazol-5-ylthio acetamido $)$ benzoate $7 \mathbf{a}(0.30 \mathrm{~g}, 0.80 \mathrm{mmol})$ in THF $(22 \mathrm{~mL})$ was added the prepared LiHMDS solution at $-78^{\circ} \mathrm{C}$. After stirring at $-78{ }^{\circ} \mathrm{C}$ for $1 \mathrm{~h}$, the mixture was heated at $80^{\circ} \mathrm{C}$ for $2 \mathrm{~h}$. The mixture was quenched with trifluoroacetic acid, and concentrated to give a crude solid, which was washed with $\mathrm{MeOH}$ to give $\mathbf{8 a}(0.09 \mathrm{~g}, 33 \%)$ as a white solid. $\mathrm{mp}: 217-219^{\circ} \mathrm{C}$ ${ }^{1} \mathrm{H}$ NMR (300 MHz, DMSO-d $) \delta 5.13(\mathrm{~s}, 2 \mathrm{H}), 7.19-7.24(\mathrm{~m}$, $3 \mathrm{H}), 7.32-7.48(\mathrm{~m}, 6 \mathrm{H}), 7.57-7.66(\mathrm{~m}, 3 \mathrm{H}), 7.94(\mathrm{~d}, 1 \mathrm{H}, J=8.1$ $\mathrm{Hz}), 11.66(\mathrm{~s}, 1 \mathrm{H}) ;{ }^{13} \mathrm{CNMR}\left(75 \mathrm{MHz}, \mathrm{DMSO}-\mathrm{d}_{6}\right) \delta 70.0,105.0$, 115.3, 116.0, 116.1, 116.3, 126.7, 126.9, 128.5, 128.7, 129.2, 137.1, 139.8, 140.8, 154.2, 160.2, 161.6, 166.5; IR 3140, 1644, $1600,1253,1165 \mathrm{~cm}^{-1}$; GC/MS(EI) $\mathrm{C}_{23} \mathrm{H}_{17} \mathrm{~N}_{5} \mathrm{O}_{3} \mathrm{~S}\left[\mathrm{M}^{+}\right] 443.1$ found 443.0.

3-[1-(4-Ethoxyphenyl)-1 H-tetrazol-5-ylthio]-4-hydroxyquinolin-2-(1H)one (8b): white solid, $\mathrm{mp}: 243-247{ }^{\circ} \mathrm{C} ;{ }^{1} \mathrm{HNMR}$ $\left(300 \mathrm{MHz}, \mathrm{DMSO}-d_{6}\right) \delta 1.33(\mathrm{t}, 3 \mathrm{H}, J=7.4 \mathrm{~Hz}), 4.04$ (q, 2H, $J=7.5 \mathrm{~Hz}), 7.11(\mathrm{~d}, 2 \mathrm{H}, J=8.9 \mathrm{~Hz}), 7.19-7.24(\mathrm{~m}, 1 \mathrm{H}), 7.30$ $(\mathrm{d}, 1 \mathrm{H}, J=8.2 \mathrm{~Hz}), 7.57-7.63(\mathrm{~m}, 3 \mathrm{H}), 7.92(\mathrm{~d}, 1 \mathrm{H}, J=7.8$ $\mathrm{Hz}), 11.65(\mathrm{~s}, 1 \mathrm{H}) ;{ }^{13} \mathrm{C} \mathrm{NMR}\left(75 \mathrm{MHz}, \mathrm{DMSO}_{-}\right) \delta 14.4,63.6$, $96.2,114.5,115.2,115.3,121.6,123.7,125.6,126.0,132.4$, 
139.0, 153.4, 159.7, 160.8, 165.6; IR 3145, 1647, 1599, 1259, $1160 \mathrm{~cm}^{-1}$; GC/MS(EI) $\mathrm{C}_{18} \mathrm{H}_{15} \mathrm{~N}_{5} \mathrm{O}_{3} \mathrm{~S}\left[\mathrm{M}^{+}\right] 381.0$ found 381.0 .

4-Hydroxy-3-[1-(4-methoxyphenyl)-1H-tetrazol-5-ylthio] quinolin-2-(1H)one (8c): white solid, $\mathrm{mp}:$ : 236-240 ${ }^{\circ} \mathrm{C} ;{ }^{1} \mathrm{HNMR}$ $\left(300 \mathrm{MHz}, \mathrm{DMSO}_{6} \mathrm{~d}_{6}\right) \delta 3.79(\mathrm{~s}, 3 \mathrm{H}), 7.14(\mathrm{~d}, 2 \mathrm{H}, J=8.9 \mathrm{~Hz})$, 7.19-7.24 (m, 1H), 7.30 (d, 1H, $J=8.2 \mathrm{~Hz}), 7.57-7.65(\mathrm{~m}, 3 \mathrm{H})$, 7.94 (d, 1H, $J=7.7 \mathrm{~Hz}), 11.65$ (s, 1H); ${ }^{13} \mathrm{C} \mathrm{NMR}$ (75MHz, DMSO-d $\left.{ }_{6}\right) \delta 96.8,115.2,115.5,115.7,116.0,122.4,122.5,126.5$, 126.8, 133.3, 139.7, 154.2, 161.1, 161.6, 166.4; IR 3353, 1647, $1600,1261,1160 \mathrm{~cm}^{-1}$; GC/MS(EI) $\mathrm{C}_{17} \mathrm{H}_{13} \mathrm{~N}_{5} \mathrm{O}_{3} \mathrm{~S}\left[\mathrm{M}^{+}\right] 367.1$ found 367.1 .

4-Hydroxy-3-[1-(4-hydroxyphenyl)-1H-tetrazol-5-ylthio] quinolin-2-(1H)one (8d): To a solution of HMDS $(2.17 \mathrm{~mL}$, $10.40 \mathrm{mmol})$ in THF $(58 \mathrm{~mL})$ was added $1.6 \mathrm{M}$ of $n-\mathrm{BuLi}(6.47$ $\mathrm{mL}, 10.40 \mathrm{mmol})$ at $-78^{\circ} \mathrm{C}$, and the mixture was stirred for $1 \mathrm{~h}$. To a solution of methyl 2-\{2-[1-(4-hydroxyphenyl)-1H-tetrazol-5-ylthio]acetamido benzoate $6(1.00 \mathrm{~g}, 2.60 \mathrm{mmol})$ in THF $(70 \mathrm{~mL})$ was added the prepared LiHMDS solution at $-78^{\circ} \mathrm{C}$. After stirring at $-78^{\circ} \mathrm{C}$ for $1 \mathrm{~h}$, the mixture was heated at $80^{\circ} \mathrm{C}$ for $2 \mathrm{~h}$. The mixture was quenched with trifluoroacetic acid, and concentrated to give a crude solid, which was washed with $\mathrm{MeOH}$ to give 8d $(0.30 \mathrm{~g}, 32 \%)$ as a white solid. mp : 220-222 ${ }^{\circ} \mathrm{C} ;{ }^{1} \mathrm{H}$ NMR (300 MHz, DMSO-d $) \delta 7.14$ (d, 2H, $\left.J=8.9 \mathrm{~Hz}\right)$, 7.19-7.24 (m, 1H), 7.30 (d, 1H, J=8.2 Hz), 7.57-7.65 (m, 3H), $7.94(\mathrm{~d}, 1 \mathrm{H}, J=7.7 \mathrm{~Hz}), 11.65(\mathrm{~s}, 1 \mathrm{H}) ;{ }^{13} \mathrm{C} \mathrm{NMR}(75 \mathrm{MHz}, \mathrm{DM}-$ SO- $\left.d_{6}\right) \delta 96.1,114.6,115.4,116.1,121.6,123.8,124.3,126.0$, 132.4, 139.0, 153.6, 159.1, 161.0, 165.9; IR 3133, 1622, 1590, $1188,1154 \mathrm{~cm}^{-1}$; GC/MS(EI) $\mathrm{C}_{16} \mathrm{H}_{11} \mathrm{~N}_{5} \mathrm{O}_{3} \mathrm{~S}\left[\mathrm{M}^{+}\right] 353.0$ found 353.3 .

3-[1-(4-Benzyloxyphenyl)-1H-tetrazol-5-ylthio]-4-methoxyquinolin-2-(1H)one (2a): To a solution of 3-[1-(4-benzyloxyphenyl)-1H-tetrazol-5-ylthio]-4-hydroxyquinolin-2-(1H)one 8a $(0.10 \mathrm{~g}, 0.20 \mathrm{mmol})$ in $\mathrm{DMF}(3 \mathrm{~mL})$ was added $\mathrm{NaH}(0.01 \mathrm{~g}$, $0.30 \mathrm{mmol})$ and Iodomethane $(0.06 \mathrm{~mL}, 1.00 \mathrm{mmol})$, and the mixture was stirred at $\mathrm{rt}$ for $3 \mathrm{~h}$. The mixture was extracted with ethyl acetate, and purified with column chromatography (ethyl acetate : hexane $=1: 4)$ to give $\mathbf{2 a}(0.09 \mathrm{~g}, 60 \%)$ as a white solid. mp: $171-174{ }^{\circ} \mathrm{C} ;{ }^{1} \mathrm{H}$ NMR $\left(300 \mathrm{MHz}, \mathrm{CDCl}_{3}\right) \delta 4.25(\mathrm{~s}, 3 \mathrm{H})$, $5.08(\mathrm{~s}, 2 \mathrm{H}), 7.07-7.12(\mathrm{~m}, 3 \mathrm{H}), 7.22(\mathrm{~d}, 1 \mathrm{H}, J=7.4 \mathrm{~Hz}), 7.34-$ $7.45(\mathrm{~m}, 5 \mathrm{H}), 7.49-7.54(\mathrm{~m}, 1 \mathrm{H}), 7.67(\mathrm{~d}, 2 \mathrm{H}, J=8.9 \mathrm{~Hz}), 7.84$ $(\mathrm{d}, 1 \mathrm{H}, J=8.1 \mathrm{~Hz}), 11.12(\mathrm{~s}, 1 \mathrm{H}) ;{ }^{13} \mathrm{C}$ NMR (75 MHz, DMSO$\left.d_{6}\right) \delta 62.4,69.7,108.4,115.5,115.6,115.7,122.3,123.4,126.0$, $126.5,127.7,128.0,128.4,132.4,136.3,138.8,152.7,159.6$, 160.3, 167.3; IR 3062, 2925, 1644, 1511, $1245 \mathrm{~cm}^{-1}$; GC/MS(EI) $\mathrm{C}_{24} \mathrm{H}_{19} \mathrm{~N}_{5} \mathrm{O}_{3} \mathrm{~S}\left[\mathrm{M}^{+}\right] 457.1$ found 457.1 .

4-Benzyloxy-3-[1-(4-ethoxyphenyl)-1H-tetrazol-5-ylthio] quinolin-2-(1H)one (2b): white solid, mp : 124-127 ${ }^{\circ} \mathrm{C} ;{ }^{1} \mathrm{H}$ NMR $\left(300 \mathrm{MHz}, \mathrm{CDCl}_{3}\right) \delta 1.42(\mathrm{t}, 3 \mathrm{H}, J=7.0 \mathrm{~Hz}), 4.03(\mathrm{q}, 2 \mathrm{H}, J=7.0$ $\mathrm{Hz}), 5.46$ (s, 2H), 6.97 (d, 2H, $J=8.9 \mathrm{~Hz}), 7.09-7.50$ (m, 8H), $7.62(\mathrm{~d}, 2 \mathrm{H}, J=8.9 \mathrm{~Hz}), 7.73(\mathrm{~d}, 1 \mathrm{H}, J=7.2 \mathrm{~Hz}), 12.39(\mathrm{~s}, 1 \mathrm{H})$; ${ }^{13} \mathrm{C} \mathrm{NMR}\left(75 \mathrm{MHz}, \mathrm{CDCl}_{3}\right) \delta 14.6,63.9,108.6,115.1,116.3$, 117.1, 122.9, 123.9, 126.1, 126.3, 128.3, 128.4, 128.7, 128.8, 132.6, 135.7, 138.5, 152.6, 160.2, 163.0, 168.1; IR 3062, 2936, $1640,1513,1249 \mathrm{~cm}^{-1}$; GC/MS(EI) $\mathrm{C}_{25} \mathrm{H}_{21} \mathrm{~N}_{5} \mathrm{O}_{3} \mathrm{~S}\left[\mathrm{M}^{+}\right] 471.1$ found 471.0 .

4-Benzyloxy-3-[1-(4-methoxyphenyl)-1H-tetrazol-5-ylthio] quinolin-2-(1H)one (2c): white solid, mp : 178-182 ${ }^{\circ} \mathrm{C} ;{ }^{1} \mathrm{HNMR}$ $\left(300 \mathrm{MHz} \mathrm{CDCl}_{3}\right) \delta 3.84(\mathrm{~s}, 3 \mathrm{H}), 5.49(\mathrm{~s}, 2 \mathrm{H}), 6.99(\mathrm{~d}, 2 \mathrm{H}, J=$ $8.7 \mathrm{~Hz}), 7.09-7.12(\mathrm{~m}, 2 \mathrm{H}), 7.37-7.54(\mathrm{~m}, 6 \mathrm{H}), 7.65$ (d, 2H, $J=$ $8.7 \mathrm{~Hz}), 7.75(\mathrm{~d}, 1 \mathrm{H}, J=8.1 \mathrm{~Hz}), 12.05(\mathrm{~s}, 1 \mathrm{H}) ;{ }^{13} \mathrm{C}$ NMR $(75$ $\left.\mathrm{MHz}, \mathrm{CDCl}_{3}\right) \delta 55.6,108.6,114.7,116.2,117.2,123.0,123.9$, $126.1,126.6,128.3,128.7,128.8,132.6,135.7,138.5,152.6$, 160.8, 163.0, 168.1; IR 3079, 2932, 1647, 1513, $1252 \mathrm{~cm}^{-1}$; GC/ $\mathrm{MS}(\mathrm{EI}) \mathrm{C}_{24} \mathrm{H}_{19} \mathrm{~N}_{5} \mathrm{O}_{3} \mathrm{~S}\left[\mathrm{M}^{+}\right] 457.1$ found 457.1 .

4-Benzyloxy-3-[1-(4-benzyloxyphenyl)-1 H-tetrazol-5-ylthio]quinolin-2-(1H)one (2d): white solid, mp : 175-179 ${ }^{\circ} \mathrm{C} ;{ }^{1} \mathrm{H}$ NMR (300 MHz, CDCl 3 ) $\delta 5.09(\mathrm{~s}, 2 \mathrm{H}), 5.47(\mathrm{~s}, 2 \mathrm{H}), 7.07(\mathrm{~d}$, $2 \mathrm{H}, J=8.7 \mathrm{~Hz}), 7.15-7.21(\mathrm{~m}, 1 \mathrm{H}), 7.26-7.53(\mathrm{~m}, 12 \mathrm{H}), 7.64(\mathrm{~d}$, $2 \mathrm{H}, J=8.8 \mathrm{~Hz}), 7.77(\mathrm{~d}, 1 \mathrm{H}, J=8.0 \mathrm{~Hz}), 11.31(\mathrm{~s}, 1 \mathrm{H}) ;{ }^{13} \mathrm{CNMR}$ $\left(75 \mathrm{MHz}, \mathrm{CDCl}_{3}\right) \delta 70.4,108.6,115.6,116.2,117.1,123.0$, $123.9,125.9,126.1,126.8,127.4,128.2,128.3,128.7,128.8$, 132.7, 135.7, 136.1, 138.5, 152.6, 160.0, 162.9, 168.1; IR 3059, $2924,1645,1512,1249 \mathrm{~cm}^{-1}$; GC/MS(EI) $\mathrm{C}_{30} \mathrm{H}_{23} \mathrm{~N}_{5} \mathrm{O}_{3} \mathrm{~S}\left[\mathrm{M}^{+}\right]$ 533.1 found 533.1 .

5,7-Dichloro-4-hydroxy-3-[1-(4-methoxy-phenyl)-1H-tetrazol-5-ylsulfanyl]-1H-quinolin-2-one (11): To a solution of HMDS (0.04 mL, $0.2 \mathrm{mmol})$ in THF $(1 \mathrm{~mL})$ was added $2.5 \mathrm{M}$ of $n$ - $\mathrm{BuLi}(0.07 \mathrm{~mL}, 0.2 \mathrm{mmol})$ at $-78^{\circ} \mathrm{C}$, and the mixture was stirred for $1 \mathrm{~h}$. To a solution of 2,4-dichloro-6-\{2-[1-(4-methoxy-phenyl)-1 $H$-tetrazol-5-ylsulfanyl]-acetylamino $\}$-benzoic acid methyl ester $(0.03 \mathrm{~g}, 0.06 \mathrm{mmol}) \mathbf{1 0}(0.03 \mathrm{~g}, 0.06 \mathrm{mmol})$ in THF $(1 \mathrm{~mL})$ was added the prepared LiHMDS solution at $-78{ }^{\circ} \mathrm{C}$. After stirring at $-78{ }^{\circ} \mathrm{C}$ for $1 \mathrm{~h}$, the mixture was heated at $80{ }^{\circ} \mathrm{C}$ for $4 \mathrm{~h}$. The mixture was quenched with trifluoroacetic acid, and concentrated to give a crude solid, which was washed with $\mathrm{MeOH}$ to give $11(0.01 \mathrm{~g}, 33 \%)$ as a white solid. $\mathrm{mp}:$ : 254$257{ }^{\circ} \mathrm{C} ;{ }^{1} \mathrm{H}$ NMR (300 MHz, DMSO- $\left.d_{6}\right) \delta 3.79$ (s, $3 \mathrm{H},-\mathrm{OCH}_{3}$ ), 7.12 (d, $J=8.9 \mathrm{~Hz}, 2 \mathrm{H}, \mathrm{ArH}), 7.29$ (d, $J=2.0 \mathrm{~Hz}, 1 \mathrm{H}, \mathrm{ArH}), 7.36$ $(\mathrm{d}, J=1.9 \mathrm{~Hz}, 1 \mathrm{H}, \mathrm{ArH}), 7.60(\mathrm{~d}, J=8.9 \mathrm{~Hz}, 2 \mathrm{H}, \mathrm{ArH}), 11.82(\mathrm{~s}$, $1 \mathrm{H},-\mathrm{NH}) ;{ }^{13} \mathrm{C}-\mathrm{NMR}\left(75 \mathrm{MHz}, \mathrm{DMSO}-d_{6}\right) \delta 25.0,55.5,66.9$, $114.2,114.8,124.4,125.7,126.1,132.3,135.9,142.0,153.2$, 160.0, 160.5; IR 3053, 2827, 1642, 1560, 1512, $1178 \mathrm{~cm}^{-1}$; GC/ $\mathrm{MS}(\mathrm{EI}) \mathrm{C}_{17} \mathrm{H}_{11} \mathrm{Cl}_{2} \mathrm{~N}_{5} \mathrm{O}_{3} \mathrm{~S}\left[\mathrm{M}^{+}\right] 435.0$ found 435.0 .

IC $_{50}$ determination. The five hits that inhibited SARS-CoV $3 \mathrm{CL}^{\text {pro }}$ at $10 \mu \mathrm{M}$ were also evaluated against CoV-229E $3 \mathrm{CL}^{\text {pro }}$, EV71 3C ${ }^{\text {pro }}$, CVB3 3C $C^{\text {pro }}$, and RV14 3C ${ }^{\text {pro }}$. In the assay solution, the activities of these proteases $(0.5 \mu \mathrm{M})$ with $10 \mu \mathrm{M}$ fluorogenic substrate Dabcyl-KTSAVLQSGFRKME-Edans in the buffers of $10 \mathrm{mM}$ MES at pH 6.5 and 6.0 (the optimal pH for EV71 and RV14 proteases, respectively) and $10 \mathrm{mM}$ Hepes at $\mathrm{pH} 7.5$ (for $\mathrm{CoV}-229 \mathrm{E}$ and CVB3 proteases) were measured in the presence of various concentrations of the inhibitors to obtain the $\mathrm{IC}_{50}$.

Computer modeling. For the modeling analysis, we used the crystal structure of SARS $3 \mathrm{CL}^{\mathrm{pro}}$ in complex with a peptide inhibitor (PDB code $1 \mathrm{UK} 4){ }^{7}$ The structures of CVB3 $3 \mathrm{C}^{\text {pro }}$ were solved by us and the structural model of EV71 $3 \mathrm{C}^{\mathrm{pro}}$ is constructed from the structure of RV $3 \mathrm{C}^{\text {pro }}$ (PDB code 1CQQ) ${ }^{8}$ Docking process was performed using an automated ligand-docking subprogram of the Discovery Studio Modeling 1.2 SBD (Accelrys, Inc., San Diego, CA), with a set of parameters chosen to control the precise operation of the genetic algorithm. Docking runs were carried out using standard default settings "grid resolution" of $5 \AA$, "site opening" of $12 \AA$ and "binding site" selected for defining the active site cavity. 


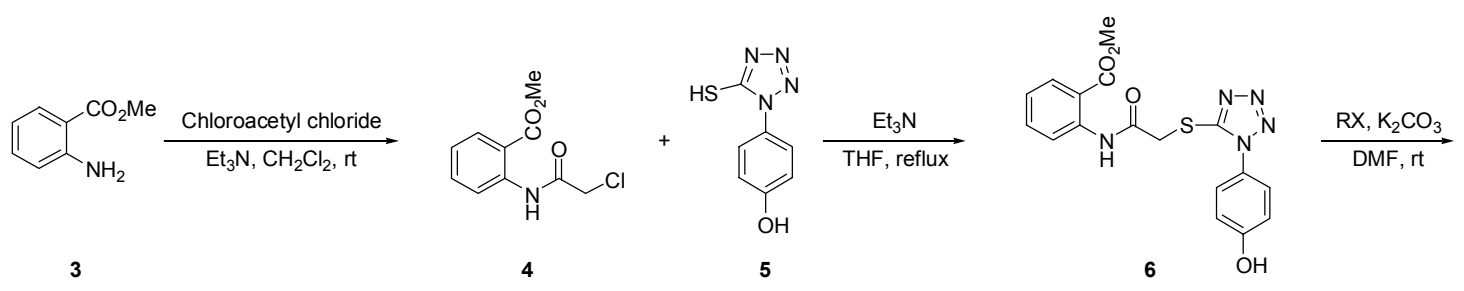<smiles>COc1ccccc1NC(=O)CSc1nnnn1-c1ccc(O)cc1</smiles>

7a $\mathrm{OR}=\mathrm{OBn}$

7c $\mathrm{OR}=\mathrm{OMe}$

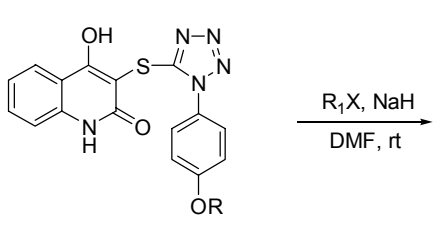

8a $\mathrm{OR}=\mathrm{OBn}$

8 b OR $=$ OEt

$8 \mathrm{c} \quad \mathrm{OR}=\mathrm{OMe}$

8d $\mathrm{OR}=\mathrm{OH}$

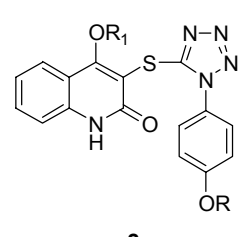

2

2a $\mathrm{OR}=\mathrm{OBn}, \mathrm{OR}_{1}=\mathrm{OMe}$

2b $\mathrm{OR}=\mathrm{OEt}, \mathrm{OR}_{1}=\mathrm{OBn}$

2c $\mathrm{OR}=\mathrm{OMe}, \mathrm{OR}_{1}=\mathrm{OBn}$ 2d $\mathrm{OR}=\mathrm{OR}_{1}=\mathrm{OBn}$

Scheme 1. Synthesis of Benzoquinolinone Derivatives

\section{Results and Discussion}

Synthesis of benzoquinolinone derivatives. We first synthesized non-chlorinated benzoquinolinone 2 because of chemical availability. The preparation of alkylated compounds $\mathbf{2}$ was described in Scheme 1. Methyl 2-aminobenzoate 3 was reacted with chloroacetyl chloride to give chloroacetyl amide 4 in $90 \%$, which was coupled with 4-(5-mercaptotetrazol-1-yl)phenol 5 to afford 6 in 27\%. Alkylation of 6 with alkyl halide yields 7 (54\% in 7a), and benzoquinolinone 8 was obtained through ring closure reaction of 7 with LiHMDS (33\% in 8a). Alkylation on the hydroxyl group of benzoquinolinone with various alkyl halides provided the benzoquinolinone derivatives 2 (60\% in 2a). Preparation of methylated dichloro-benzoquinolinone 11 was started from methyl 2-amino-4,6-dichlorobenzoate 9, which was followed by the same synthetic root as for the preparation of $7 \mathbf{a}$ and $\mathbf{8 a}$.

Inhibitory activities of the compounds. As a non-chlorinated analog of the benzoquinolinone 1, compound $\mathbf{8 d}$ and its analogs $(\mathbf{8 a - 8 c}, \mathbf{2 a - 2 d})$ as well as dichlorinated analog 11 were prepared. $\mathrm{IC}_{50}$ values of these newly prepared compounds $(\mathbf{2}, \mathbf{8}, \mathbf{1 1})$ in inhibiting SARS-CoV $3 \mathrm{CL}^{\text {pro }}$ were determined (Table 1). Among the mono-alkylated benzoquinolinone 8, 8a which has benzyl group as R showed higher inhibitory potency than $\mathbf{8 b}$ $(\mathrm{R}=$ ethyl $)$ or $8 \mathrm{c}(\mathrm{R}=$ methyl $)$. In di-alkylated benzoquinolinone $\mathbf{2}, \mathbf{2} \mathbf{a}$ is the most active compound. Compound $\mathbf{2} \mathbf{a}$ also has benzyl group as $\mathrm{R}$ and methyl group as $\mathrm{R}_{1}$. Thus compounds $\mathbf{8 a}$ and $\mathbf{2 a}$ showed more than $50 \%$ inhibition of the enzyme activity at 12.5 $\mu \mathrm{M}$, and their $\mathrm{IC}_{50}$ values are $10.0 \mu \mathrm{M}$ and $2.0 \mu \mathrm{M}$, respectively. Even though we prepared and synthesized limited number of benzoquinoline compounds, we confirmed that computer modeling analysis led us to a direction for discovery of more active inhibitors. Base on this molecular modeling study, we designed and synthesized alkylated benzoquinolines at both sites of the hydroxyl groups. Thus we found the compound $\mathbf{2 a}$ showed five times higher inhibiting activity compared to the compound $\mathbf{1}$.

We further tested the prepared benzoquinolinones to EV71
Table 1. Inhibition Potency of the Benzoquinolinones to SARS-CoV $3 \mathrm{CL}^{\text {pro }}$, EV71 $3 \mathrm{C}^{\text {pro }}$ and CVB3 $3 C^{\text {pro }}$

\begin{tabular}{|c|c|c|c|c|}
\hline \multirow[b]{2}{*}{ Compound } & \multicolumn{3}{|c|}{$\mathrm{IC}_{50}(\mu \mathrm{M})$} & \\
\hline & $\begin{array}{l}\text { SARS } \\
3 \mathrm{CL}^{\text {pro }}\end{array}$ & $\begin{array}{l}\text { EV71 } \\
3 C^{\text {pro }}\end{array}$ & $\begin{array}{l}\text { CVB3 } \\
3 C^{\text {pro }}\end{array}$ & \\
\hline 1 & 10 & $>12.5$ & $>12.5$ & \\
\hline $2 a$ & 2 & $>12.5$ & $>12.5$ & $\mathrm{R}=\mathrm{Bn}, \mathrm{R}_{1}=\mathrm{Me}$ \\
\hline $2 \mathbf{b}$ & $>12.5$ & $>12.5$ & $>12.5$ & $\mathrm{R}=\mathrm{Et}, \mathrm{R}_{1}=\mathrm{Bn}$ \\
\hline $2 c$ & $>12.5$ & $>12.5$ & $>12.5$ & $\mathrm{R}=\mathrm{Me}, \mathrm{R}_{1}=\mathrm{Bn}$ \\
\hline 2d & $>12.5$ & $>12.5$ & $>12.5$ & $\mathrm{R}=\mathrm{Bn}, \mathrm{R}_{1}=\mathrm{Bn}$ \\
\hline $8 a$ & 10 & $>12.5$ & $>12.5$ & $\mathrm{R}=\mathrm{Bn}, \mathrm{R}_{1}=\mathrm{H}$ \\
\hline $8 b$ & $>12.5$ & $>12.5$ & $>12.5$ & $\mathrm{R}=\mathrm{Et}, \mathrm{R}_{1}=\mathrm{H}$ \\
\hline $8 c$ & $>12.5$ & $>12.5$ & $>12.5$ & $\mathrm{R}=\mathrm{Me}, \mathrm{R}_{1}=\mathrm{H}$ \\
\hline $8 d$ & $>12.5$ & $>12.5$ & $>12.5$ & $\mathrm{R}=\mathrm{H}, \mathrm{R}_{1}=\mathrm{H}$ \\
\hline 11 & $>12.5$ & $>12.5$ & $>12.5$ & \\
\hline
\end{tabular}

$3 C^{\text {pro }}$ and CVB3 $3 C^{\text {pro }}$, however none of the compounds showed more than $50 \%$ inhibition of the enzyme activities at $12.5 \mu \mathrm{M}$.

Computer modeling of 2a binding with SARS 3CL ${ }^{\text {pro }}$. Compared to the binding mode of 22723 (1) as shown in the left panel of Figure 2, 2a binds in the SARS 3CL ${ }^{\text {pro }}$ active site with its benzyl group extended into S3 site. Although from the Figure 2, 1 can form an H-bond with the protease, but this H-bond is removed in the 2a-3CLpro interactions. The extra side chains in 2a can form several other interactions such as hydrophobic inter- 
action with the protease. In this area, Met165, Leu167, and Pro168 can provide hydrophobic interactions. This can be revealed by the better binding score (82.7) for 2 a compared to that (72.1) for $\mathbf{1}$. This extra interaction of the compound $\mathbf{2 a}$ with $\mathrm{S} 3$ site of the protease increases its binding affinity and reduces its $\mathrm{IC}_{50}$ value by 5 -fold (from $10 \mu \mathrm{M}$ for 1 to $2 \mu \mathrm{M}$ for $\mathbf{2 a}$ ). This binding mode is actually similar to that of 43146 with SARS $3 \mathrm{CL}^{\text {pro }}$ as reported previously. ${ }^{5}$

Acknowledgments. This work was supported by a grant from Korea Research Institute of Chemical Technology.

\section{References}

1. Ksiazek, T. G.; Erdman, D.; Goldsmith, C. S.; Zaki, S. R.; Peret, T.; Emery, S.; Tong, S.; Urbani, C.; Comer, J. A.; Lim, W.; Rollin, P. E.; Dowell, S. F.; Ling, A. E.; Humphrey, C. D.; Shieh, W. J.; Guarner, J.; Paddock, C. D.; Rota, P.; Fields, B.; DeRisi, J.; Yang, J. Y.; Cox, N.; Hughes, J. M. N. Eng. J. Med. 2003, 348, 1953-1966.

2. Liang, P. H. Curr. Top Med. Chem. 2006, 6, 361-376.

3. Kuo, C. J.; Liu, H. G.; Lo, Y. K.; Seong, C. M.; Lee, K. I.; Jung, Y.
S.; Liang, P. H. FEBS Lett. 2009, 583, 549-555.

4. De Clercq, E. Expert Rev. Anti Infect. Ther. 2006, 4, 291-302.

5. Zhai, S.; Liu, W.; Yan, B. Recent Patents Anti-Infect Drug Disc. 2007, 2, 1-10.

6. Melnick, J. L. Enterovirus: Polioviruses, Coxsackieviruses, Echoviruses, and Newer Enteroviruses In Virology, $3^{\text {rd }}$ ed.; LippincottRaven: Philadephia, U.S.A. 1996.

7. Krausslich, H. G.; Wimmer, E. Annu. Rev. Biochem. 1998, 57, 701754.

8. Kuo C. J.; Shie, J. J.; Fang, J. M.; Yen, G. R.; Hsu, J. T. A.; Tseng, S. N.; Chang, S. C.; Lee, C. Y.; Shih, S. R.; Liang, P. H. Bioorg. Med. Chem. 2008, 16, 7388-7398.

9. Kuo, C. J.; Chi, Y. H.; Hsu, J. T. A.; Liang, P. H. Biochem. Biophys. Res. Commun. 2004, 318, 862-867.

10. Yang, H.; Yang, M.; Ding, Y.; Liu, Y.; Lou, Z.; Zhou, Z.; Sun, L.; Mo, L.; Ye, S.; Pang, H.; Gao, G. F.; Anard, K.; Bartlam, M.; Hilgenfeld, R.; Rao, Z. Proc. Natl. Acad. Sci. U.S.A. 2003, 100, 1319013195.

11. Matthews, D. A.; Dragovich, P. S.; Webber, S. E.; Fuhrman, S. A.; Patick, A. K.; Zalman, L. S.; Hendrickson, T. F.; Love, R. A.; Prins, T. J.; Marakovits, J. T.; Zhou, R.; Tikhe, J.; Ford, C. E.; Meador, J. W.; Ferre, R. A.; Brown, E. L.; Binford, S. L.; Brothers, M. A.; Delisle, D. M.; Worland, S. T. Proc. Natl. Acad. Sci. U.S.A. 1999, 96, 11000-11007. 